\title{
The Application of "Internet Plus Nursing Service" Mode under the Epidemic of Novel Coronavirus Pneumonia
}

\author{
Lin Wang1*, Ruiying Huang1, Zhili Chen' ${ }^{1}$, Caixiang Zhang1, \\ Yanying Zhu' ${ }^{1}$, Meijuan Luo', Wanping Xu' ${ }^{1}$, Weiju Chen ${ }^{2} \#$ \\ ${ }^{1}$ Transitional Care Department, The First Affiliated Hospital of Jinan University, Guangzhou, China \\ ${ }^{2}$ School of Nursing, Jinan University, Guangzhou, China \\ Email: wanglin18903078509@126.com, ${ }^{*}$ chenweiju@126.com
}

How to cite this paper: Wang, L., Huang, R. Y., Chen, Z. L., Zhang, C. X., Zhu, Y. Y., Luo, M. J., Xu, W. P., \& Chen, W. J. (2020). The Application of "Internet Plus Nursing Service" Mode under the Epidemic of Novel Coronavirus Pneumonia. Open Journal of Social Sciences, 8, 255-264.

https://doi.org/10.4236/jss.2020.85018

Received: April 29, 2020

Accepted: May 19, 2020

Published: May 22, 2020

Copyright $\odot 2020$ by author(s) and Scientific Research Publishing Inc. This work is licensed under the Creative Commons Attribution International License (CC BY 4.0).

http://creativecommons.org/licenses/by/4.0/

\begin{abstract}
Objective: To explore the application of "Internet plus nursing service" program under the pandemic of novel coronavirus pneumonia (NCP). Methods: Nurses of Transitional Care Department of the author's hospital assessed and categorized adequately the patients who applied for services online and used a combination of online and offline approaches to provide nursing care to patients at home. Results: During the pandemic of novel coronavirus pneumonia from January $19^{\text {th }}$ to March $31^{\text {st }}$, Transitional Care Department adopted the "Internet plus nursing service" mode to provide nursing services for 357 cases of the in home patients, which was approximately three times the number of the same period in last year. As a result of a comprehensive evaluation, 248 cases of patients' medical care problems were solved through online educational guidance and consultation; 101 cases of patients were served by home-based services; and 8 cases of patients were recommended to receive professional medical treatment in hospital. There were no patients admitted to the hospital for reasons such as changes in their conditions through follow-up visits, and home visit nurses were in good health. Conclusion: In-home patients have greater demands for the "Internet plus nursing service" due to the pandemic of novel coronavirus pneumonia. Applying the "Internet plus nursing service" mode to serve patients can not only meet the medical needs of in-home patients, but also effectively relieve the pressure of hospital treatment. Moreover, it also reduces the risk of cross-infection by decreasing the number of staffs for mass gatherings, which plays a paramount role in control and prevention of the novel coronavirus pneumonia prevention.
\end{abstract}




\section{Keywords}

Internet Plus Nursing Service, Novel Coronavirus Pneumonia, Pandemic

\section{Introduction}

In late 2019, with the outbreak of novel coronavirus pneumonia (COVID-19), China has initiated the first-level response to the major public health emergency (CCTV News Client, 2020). Chinese Center for Disease Control and Prevention requested the public to avoid unnecessary travel and stay away from crowded places. Hospital visits are not recommended for non-critical patients. In order to meet the medical needs of the public, National Health Commission in China suggested that the medical institutions could make good use of Internet plus healthcare to supply the public with high-quality and convenient medical consultation services under the pandemic situation (Medical Administration Bureau in China, 2020a). "Internet plus nursing service" mainly refers to the use of registered nurses in medical institutions, relying on information technology with "online application, offline service" mode, to provide nursing services for the homebound patients or the post-discharged patients (National Health Commission in China, 2019). Our hospital set up a Transitional Care Department with a manager and nine full-time nurses in different specialties in 2007. The extensional nursing service includes home visits for geriatric patients with chronic diseases, wound and stormy care, maternal and child health care, and follow-up care of cancer (Chen, Lin, \& Zhai, 2017). As one of the pilot medical institutions of "Internet plus nursing service" in Guangdong Province, our hospital has actively responded to the national policy and accumulated abundantly practical experience. During the pandemic of the NCP, the hospital still insisted on providing the "Internet plus Nursing Service" and formulated a series of countermeasures, which achieved pleasant results.

\section{Methods}

\subsection{Participants}

The study population for the application of the "Internet plus nursing service" mode was selected from the patients at their residency who were served by Transitional Care Department under the pandemic situation from January $19^{\text {th }}$ to March $31^{\text {st }}$ in 2020. It mainly consists of elderly patients, disabled or semi-disabled people, and people with limited mobility such as recovering patients, terminal patients, and chronically ill patients. Besides, maternal and infant are also included in the study population (Guangdong Provincial National Health Commission in China, 2019). 


\subsection{Procedures}

\subsubsection{Application of the "Wechat Mini Program" as an Online Platform Tool}

The information technology platform for the "Internet plus nursing service" is a WeChat mini program which is an application that can be used without downloading and was developed and put into use in the early 2019. The main functions of this mini program are consultation, application for home visiting services and health education. Patients can directly make an online appointment through the WeChat mini program by scanning a quick response code (QR code) or by using searching function. After reading and checking the box of the informed consent, patients can request a home visiting.

\subsubsection{Classification of Service Items and Cases for Home Visits}

Before the pandemic of COVID-19, the "Internet plus nursing service" was conducted by the mode of online appointments and offline home visiting to provide nursing service to the patients. However, in the current COVID-19 pandemic, it is necessary to follow the prevention instructions in order to reduce the risk of cross-infection by minimizing face-to-face contact between the patients and nurses. Therefore, according to the principle of differentiation of service items, the hospital mainly affords online health education guidance and consultation for the patients who apply for non-skill nursing service; for those who apply for skill nursing service items are fully evaluated to provide home visits based on their conditions.

\subsubsection{Formulation of the "Internet Plus Nursing Service" Process Applied in COVID-19 Pandemic}

The procedures of the "Internet plus Nursing Service" are listed below: 1) Making a comprehensive assessment to the applicant, including health status, past medical history, psychological condition, home condition, and healthcare need, etc. 2) Providing the patients with appropriate services based on the results of the assessment, containing online education guidance and offline home visits services. 3) For the patients applying for home visits services, the nurses are required to use telephone to assess the patients and their family members by the COVID-19 epidemiological questionnaire which is composed of demographic information, epidemiological history and suspected symptoms of NCP. If patients have history of exposure or suspicious symptoms of COVID-19 are guided to hospitals for treatment as soon as possible. 4) Following up the condition of the patients on the first, third and seventh days after service.

\subsubsection{Formulation of the Home Visits Process Applied in COVID-19 Pandemic}

The formulation focused on redefining the home visits service process under the COVID-19 pandemic. 1) Prior to providing home visits, the nurses are required to assess the pandemic exposure history of the patients and their family members by the COVID-19 epidemiological questionnaire. Secondly, the pandemic 
situation around the patient's community is evaluated. Thirdly, briefly explain the risk of home visit services to the patients and their family. Last but not least, the medical personnel should bring all protective equipment in accordance with the principles of pandemic protection. Before home visits, insure all documents are prepared such as identity cards and working cards in order to make a registration when entering the patient's community. 2) Home visit nurses should precede hand hygiene upon arrival at the patient's home, then wearing face masks, protective clothing, goggles, shoe covers and gloves at once. After all the protective measures are done, nurses instruct patients and their family to wear face masks correctly and inform them to maintain at least one meter between nurses and do not gather around the personnel when operating nursing service (Medical Administration Bureau in China, 2020b). The COVID-19 epidemiological questionnaire is examined again with the patients and their family by signing the form to confirm all the information is correct. Further confirmation for the content of the nursing service as well as its risk with the patient is necessary. Before the nursing service is conducted, the patients and their family are required to sign the informed consent form for home visit. Next, the conditions of the patients are fully assessed and nursing service is carried out in accordance with the operation procedures. At the end of the home visits, medical waste is placed in yellow garbage bags and hand hygiene is proceeding. Health education and guidance on home protection under the pandemic situation are supported by home visit nurses. The medical waste is brought back to the hospital and disposed according to the medical waste regulations.

\subsubsection{Skills and Knowledge Training for Nurses Related to the COVID-19 Pandemic}

The home visit nurses were trained for getting familiar with the home visits service procedures and learning knowledge related to the COVID-19 such as prevention and control policy, scheme of treatment, personal protection, psychological crisis intervention, occupational exposure management process, fundamental pandemic prevention and public health education through self-learning, group discussion and hospital training. Processes of the "Internet plus nursing service" applied in COVID-19 pandemic and that of the home visits in COVID-19 pandemic are instructed through the group discussion and practice. Last but not least, the nurses were trained on proper wearing of protective suits.

\subsection{Evaluation Index}

The number of cases served by the "Internet plus nursing service" from January $19^{\text {th }}$ to March $31^{\text {st }}, 2020$, was collected. Follow-up visit was implemented to monitor the conditions of the patients. Moreover, health conditions of the nurses were also monitored everyday by collecting their health information.

\section{Results}

1) There were a total of 357 cases of in-home patients accepted online nursing 
consultation in which 113 cases were male and 244 cases were female. The study group was mainly comprised of maternity group and geriatric group with chronic illnesses, where the maternity group occupied 127 cases, aged 24 to 43 with an average of $30.72 \pm 4.07$ year old; the geriatric group took up 220 cases, aged 60 to 97 with an average of $81.07 \pm 9.17$ year old. The rest of the 10 cases of patients were from other group. Participants' demographic characteristics were presented in Table 1.

2) From January $19^{\text {th }}$ to March $31^{\text {st }}, 2020$, a total of 357 cases of patients at home were provided nursing service by Transitional Care Department, which was approximately three times as many as the same period time in last year. After classification, there were 248 cases of patients requiring online education and guidance, while 101 cases of patients asking for home visits nursing service. The remaining 8 cases in which patients were directed to the hospital were due to serious illness or fever. There were 18 service items provided within the "Internet plus nursing service", and the total number of services was 376 in terms of service items. Service items and cases were shown in Table 2.

3) With the exception of the 8 patients who were guided to the hospital for a more professional medical treatment, the health problems of the remaining 349 patients were effectively resolved through the provision of online educational guidance as well as home visits. There was no case of the patients visiting hospital after a one-week follow-up service.

4) Health information was collected by daily health monitoring of visiting nurses, all nurses were in good health.

Table 1. Demographic characteristics and mode of service of participants $(n=357)$.

\begin{tabular}{lcc}
\hline \multicolumn{1}{c}{ Items } & Number $(\mathrm{n})$ & $(\%)$ \\
\hline Groups & 127 & 35.57 \\
$\quad$ Geriatric group & 220 & 61.63 \\
$\quad$ Maternity group & 10 & 2.80 \\
$\quad$ Other group & 113 & \\
Gender & 244 & 31.65 \\
$\quad$ Male & & 68.35 \\
$\quad$ Female & 230 & \\
Age (year) & 127 & 64.43 \\
$<60$ & & 35.57 \\
$\geq 60$ & 248 & 28.29 \\
Service mode & 101 & 2.24 \\
Online education & 8 & 69.47 \\
Home visits & & \\
Visiting hospital & & \\
\hline
\end{tabular}


Table 2. Service items and service cases of "Internet plus nursing service" of the COVID-19 pandemic.

\begin{tabular}{|c|c|c|c|c|}
\hline Services items & $\begin{array}{l}\text { Cases of online } \\
\text { education }(n, \%)\end{array}$ & $\begin{array}{l}\text { Cases of home } \\
\text { visits }(\mathrm{n}, \%)\end{array}$ & $\begin{array}{l}\text { Cases of hospital } \\
\text { visiting }(n, \%)\end{array}$ & $\begin{array}{l}\text { Total } \\
(\mathrm{n}, \%)\end{array}$ \\
\hline Maternal and infant care & $118(31.38)$ & $8(2.13)$ & $1(0.27)$ & $127(33.78)$ \\
\hline $\begin{array}{l}\text { Retention/replacement of } \\
\text { nasal gavages care }\end{array}$ & $3(0.80)$ & $65(17.29)$ & l & $68(18.09)$ \\
\hline $\begin{array}{l}\text { Retention/replacement of } \\
\text { urethral tube care }\end{array}$ & $3(0.80)$ & $23(6.12)$ & $2(0.53)$ & $28(7.45)$ \\
\hline Pressure ulcer care & $3(0.80)$ & $13(3.46)$ & $4(1.06)$ & $20(5.32)$ \\
\hline Sputum aspiration & $3(0.80)$ & $9(2.39)$ & l & $12(3.19)$ \\
\hline PICC tube maintenance & $2(0.53)$ & $9(2.39)$ & l & $11(2.92)$ \\
\hline Intravenous blood collection & l & $8(2.13)$ & l & $8(2.13)$ \\
\hline Nasogastric feeding care & $20(5.32)$ & l & l & $20(5.32)$ \\
\hline Constipation care & $15(3.99)$ & l & l & $15(3.99)$ \\
\hline $\begin{array}{l}\text { Oral medicine } \\
\text { administration }\end{array}$ & 15 (3.99) & l & l & $15(3.99)$ \\
\hline $\begin{array}{l}\text { Blood glucose } \\
\text { management }\end{array}$ & $12(3.19)$ & l & / & $12(3.19)$ \\
\hline $\begin{array}{l}\text { Care of incontinent } \\
\text { dermatitis }\end{array}$ & $10(2.66)$ & l & l & $10(2.66)$ \\
\hline Safety education & $10(2.66)$ & l & l & $10(2.66)$ \\
\hline Vital signs management & $5(1.33)$ & l & l & $5(1.33)$ \\
\hline Diabetic foot care & $5(1.33)$ & I & I & $5(1.33)$ \\
\hline Training of ADL & $5(1.33)$ & l & l & $5(1.33)$ \\
\hline Hospice care & $3(0.80)$ & l & l & $3(0.80)$ \\
\hline $\begin{array}{l}\text { Care of tracheotomy } \\
\text { tubes }\end{array}$ & $1(0.27)$ & l & $1(0.27)$ & $2(0.53)$ \\
\hline Total & $233(61.97)$ & $135(35.90)$ & $8(2.13)$ & $376(100)$ \\
\hline
\end{tabular}

\section{Discussion}

\subsection{The Demands for the "Internet Plus Nursing Service" Is Dramatically Increased during the COVID-19 Pandemic. It Plays a Crucial Role in Pandemic Prevention and Control}

During the COVID-19 pandemic, according to principle of the Chinese Center for Disease Control and Prevention, the public should avoid unnecessary travel and stay away from crowded places to reduce the risk of cross-infection. As a result, more and more people with limited mobility and chronic ill patients access a safer and more convenient approach to acquire for medical care. As a new mode of nursing service, the "Internet plus nursing service" can address the needs of patients at home efficiently (Li, Huang, Xu, Zhang, \& Fu, 2019). Between January $19^{\text {th }}$ and March $31^{\text {st }}, 2020$, a total of 357 cases of patients were 
served, which was approximately three times as many as the same period time in last year. The COVID-19 pandemic has promoted more and more in-home patients to select the "Internet plus nursing service" to solve their medical problems. The "Internet plus nursing service" can not only reduce the inconvenience of patients travelling to the hospital, but also save time for patients waiting in line for consultation. More importantly, it actively responses the policy of $\mathrm{Na}$ tional Health Commission, reducing the risk of patients cross-infection caused by visiting hospital. It is an appropriate way of nursing service and sufficiently demonstrates its advantages during the COVID-19 pandemic.

\subsection{The Development of the "Internet Plus Nursing Service" Fulfill the Demands of Major Group of People in the COVID-19 Pandemic}

National Health Commission in China proposed that the management of medical service should be strengthened to ensure for the major group of people such as maternity, children, and the elderly. According to data from the Chinese National Bureau, the number of population aged over 60 had reached 249 million at the end of 2018 , accounting for $17.9 \%$ of the total population in China $(\mathrm{Li}$, 2019). Additionally, with the aging of the Chinese population, the incidence of chronic diseases is increasing gradually. As a result, the number of the elderly living with diseases is accelerating dramatically, so is the demand for medical care services. On January $28^{\text {th }}, 2020$, Health Sectary For The Elderly of National Health Commission pointed out that Chinese geriatric population is a high-risk and susceptible group of infectious diseases (Health Sectary for the Elderly of National Health Commission, 2020), each region should take good care of the elderly in terms of managing their health through making good use of the media and network. During the COVID-19 pandemic, we have provided services for the 220 cases of geriatric patients who applied for home visits services and their service items by adopting a combination of online health educational guidance and offline home visits services. In addition to the geriatric group, another main group is maternal and infant. Due to the rapid alternation in social roles and postpartum weakness, physical stress and changes in endocrine environment, there are many health risks exist around the postpartum female and infant so that affect their health seriously ( $\mathrm{Li}, \mathrm{Xu}, \mathrm{Tan}, \mathrm{Zhu}$, \& Zeng, 2017). During the COVID-19 pandemic, we provided service for 127 cases of maternal and infant online, and online educational guidance and consultation have effectively tackled their problems and avoided the risk of cross-infection due to hospital visiting.

\subsection{Provide Offline Home Visits Service Requires the Enhancement of Nurse Training, Well Prepared for the Assessment and Personal Protection to Ensure Personal Safety}

The COVID-19 is a highly contagious disease, mainly transmitted by respiratory 
droplet and close contact, and the population is generally susceptible (Medical Administration Bureau in China, 2020d). The notice about online medical consultation services announced by the Chinese National Health Commission (Disease Prevention and Control Bureau, 2020) states that proper protection of medical personnel is a significant measure to prevent and reduce infection among medical personnel. The notice on further strengthening the management of socially-operated medical services to prevent and control the COVID-19 epidemic (Medical Administration Bureau in China, 2020c), issued by Medical Administration Bureau in China required patients to be inquired about their epidemiological history of COVID-19 pandemic. Therefore, according to the prevention and control of the COVID-19 pandemic, we developed the process of "Internet plus nursing service" and the process of home visits service. On one hand, organizing nurses to conduct training on the knowledge, skills and service process related to the protection of COVID-19 is to ensure that the nurses are able to operate in a standardized manner and well-protected. On the other hand, using the epidemiological history questionnaire to conduct the "dual assessment" approach, that is, the safety of both parties is guaranteed by checking the epidemiological history of COVID-19 with the patients and family before and after the home visits. Through follow-up service, 101 cases of patients and families provided with home visits were in good condition, and all nurses were in good condition, too.

\section{Conclusion}

In 2019, the Chinese National Health Commission proposed the implementation plan for the pilot work of "Internet plus nursing service" (National Health Commission in China, 2019), aiming at the people's health, innovating nursing service modes, expanding service supply, and accurately meeting the demands of diverse and multi-level health needs of the people. Under the COVID-19 pandemic situation, providing nursing services for the patients using the "Internet plus nursing service" is in line with the use of the Internet diagnosis and treatment consulting services advocated by the State. Furthermore, the enforcement of the "Internet plus nursing service" conducts a comprehensive assessment of the patients and provides services to the patients in a variety of ways. It could not only fulfill the needs of homebound patients suffering from the disease, but also effectively relieve the burden of prevention and control of medical institutions. It also reduces the risk of cross-infection of patients and their families while traveling to hospital, which plays a vital role in prevention and control of the COVID-19 pandemic. Currently, COVID-19 is spreading all over the world. In order to control the pandemic, the "Internet plus nursing service" mode is worthy of reference by peers.

\section{Acknowledgements}

This study was supported by grants from Guangdong Science and Technology Research Foundation (Grant NO. 2016A0101505009). 


\section{Conflicts of Interest}

The authors declare no conflicts of interest regarding the publication of this paper.

\section{References}

CCTV News Client (2020). Pneumonia Outbreak 24 Hours: Work Leading Group of the CPC Central Committee Set Up to Deal with Epidemic 30 Provinces and Autonomous Regions Have Launched a Public Health Incident Response. http://m.news.cctv.com/2020/01/25/ARTIVdXxhUzobSvozuL6cQ44200125.shtml

Chen, W. J., Lin, Q. R., \& Zhai, C. Q. (2017). Exploration on the Mode of Transitional Care Practice and Reflection on the Future Development Trend. China Nursing Management, 17, 444-448.

Disease Prevention and Control Bureau (2020). The Notice of Prevention and Control Plan of Novel Coronavirus Pneumonia Issued by Nation Health Commission of the People's Republic of China (Fourth Edition).

http://www.nhc.gov.cn/yzygj/s7659/202002/75c6e88ecbeb42a9a26acb538383e2fc.shtml

Guangdong Provincial National Health Commission in China (2019). The Notice of 'Internet plus Nursing Service' Pilot Program to Be Carried Out in Guangdong Province, China. http://wsjkw.gd.gov.cn/gkmlpt/content/2/2268/post_2268434.html

Health Sectary for the Elderly of National Health Commission (2020). Notice on Prevention and Control of Coronavirus Disease 2019 of the Elderly. http://www.nhc.gov.cn/lljks/tggg/202001/96e82ba8a14d41b283da990d39771493.shtml

Li, L. D., Xu, X. N., Tan, Q. X., Zhu, W. P., \& Zeng, Y. E. (2017). Investigation and Analysis of Community Postpartum Women's Demand for Health Knowledge. Chinese Medical Science, 7, 51-54.

Li, X. R. (2019). Steady Growth of Population and Steady Improvement of Urbanization. http://www.stats.gov.cn/tjsj/sjjd/201901/t20190123_1646380.html

Li, Y., Huang, Y., Xu, R.J., Zhang, J., \& Fu, A. D. (2019). Application of "Internet plus Nursing Service" Mode with Hospital in Transitional Care of Discharged Patients. China Clinical Nursing, 11, 185-188.

Medical Administration Bureau in China (2020a). The Notice about Online Medical Consultation Services Issued by the National Health Committee in Epidemic Prevention and Control.

http://www.nhc.gov.cn/yzygj/s7653p/202002/ec5e345814e744398c2adef17b657fb8.sht $\mathrm{ml}$

Medical Administration Bureau in China (2020b). The Notice of the Office of the National Health and Health Committee on Guidelines for Prevention and Control of Infection in Home Isolation Medicine of Pneumonia in the Novel Coronavirus Pneumonia (Trial).

http://www.nhc.gov.cn/yzygj/s7659/202002/cf80b05048584f8da9b4a54871c44b26.shtml

Medical Administration Bureau in China (2020c). The Notice on Further Strengthening the Management of Social Hospitals to Do Health Management in the Prevention and Control of COVID-19 Epidemic.

http://www.nhc.gov.cn/yzygj/s7655/202002/2728357d4f6c4912baaf27b6302ec232.shtml

Medical Administration Bureau in China (2020d). The Notice on the Issuance of COVID-19 Treatment Programme (Trial Sixth Edition).

http://www.nhc.gov.cn/yzygj/s7653p/202002/8334a8326dd94d329df351d7da8aefc2.sht $\underline{\mathrm{ml}}$ 
National Health Commission in China (2019). 'Internet plus Nursing Services' Pilot Work Plan.

http://www.nhc.gov.cn/yzygj/s7657g/201902/bf0b25379ddb48949e7e21edae2a02da.sht $\underline{\mathrm{ml}}$ 